Running head: MERE EXPOSURE EFFECT IN AD

\title{
POSTPRINT
}

\section{Normal mere exposure effect with impaired recognition in Alzheimer's disease}

\author{
Sylvie Willems ${ }^{1}$, Stéphane Adam ${ }^{2,3}$, and Martial Van der Linden ${ }^{1,2}$ \\ ${ }^{1}$ University of Geneva, Switzerland \\ ${ }^{2}$ University of Liège, Belgium \\ ${ }^{3}$ University of Louvain-la-Neuve, Belgium
}

Address correspondence to:

Sylvie Willems

Cognitive Psychopathology Unit

Bv. Pont d'Arve, 40

CH-1205 Geneva

Switzerland

Telephone: +41-22-705-93-43

FAX: +41-22-705-93-59

e-mail: sylvie.willems@pse.unige.ch 


\begin{abstract}
We investigated the mere exposure effect and the explicit memory in Alzheimer's disease (AD) patients and elderly control subjects, using unfamiliar faces. During the exposure phase, the subjects estimated the age of briefly flashed faces. The mere exposure effect was examined by presenting pairs of faces (old and new) and asking participants to select the face they liked. The participants were then presented with a forced-choice explicit recognition task. Controls subjects exhibited above-chance preference and recognition scores for old faces. The AD patients also showed the mere exposure effect but no explicit recognition. These results suggest that the processes involved in the mere exposure effect are preserved in AD patients despite their impaired explicit recognition. The results are discussed in terms of Seamon et al.'s proposal (1995) that processes involved in the mere exposure effect are equivalent to those subserving perceptual priming. These processes would depend on extrastriate areas which are relatively preserved in $\mathrm{AD}$ patients.
\end{abstract}


Normal mere exposure effect with impaired recognition in Alzheimer's disease

The mere exposure effect refers to the increase of positive attitude which results from the repeated exposure to previously novel stimuli (Zajonc, 1968). This effect has proven to be a robust, reliable phenomenon and has been shown on a variety of stimuli (including nonsense words, line drawings, ideographs, faces, possible and impossible three-dimensional objects, melodies etc.), procedures (e.g., forced-choice preference judgments, liking ratings, pleasantness ratings, behavioural indices of stimulus preference) and populations (for reviews, see Bornstein, 1989; Harrison, 1977). In particular, a number of neuropsychological studies have demonstrated a normal mere exposure effect in amnesic or prosopagnosic patients despite a profound inability to recognize the exposed stimuli (Johnson, Kim and Risse, 1985; Johnson and Multhaup, 1992; Greve and Bauer, 1990; see however Redington, Volpe and Gazzaniga, 1984, for contradictory results). These findings offer a strong argument for postulating that the mere exposure effect is an implicit memory manifestation, independent of explicit memory (Kihlstrom, Mulvaney, Tobias et al., 2000).

Similarly, two studies have explored the mere exposure effect in Alzheimer's disease (AD). Winograd, Goldstein, Monarch et al. (1999) found that a mere exposure effect for unfamiliar faces was present in mild to moderate AD patients, although they exhibited impaired recognition memory compared to controls. More recently, Halpern and O'Connor (2000) failed to observe a mere exposure effect for unfamiliar melodies. As the earlier study used the visual modality for stimuli exposition, the authors hypothesised that implicit memory for auditory information, especially nonverbal material, may be defective in AD due to neural degeneration in auditory areas in the temporal lobes. However, Verfaillie, Keane and Johnson (2000) recently found a normal priming effect in AD patients in the auditory perceptual identification of words, despite impaired recognition. As suggested by Verfaillie et al., this discrepancy within the same modality between intact implicit memory for words and impaired 
implicit memory for melodies may reflect a dissociation between an intact auditory wordform system and an impaired melodic processing system. These results also suggest that AD partients' implicit memory performance might not depend exclusively on the modality of presentation, but also on the type of stimuli.

Besides this modality difference, other differences make both studies hardly comparable. First, different types of preference measures were used: a forced-choice preference judgment between an exposed and a novel stimulus in Winograd's et al. (1999) study and a bipolar rating scale (with the endpoints labelled least pleasant ' 1 ' and most pleasant ' 5 ') in that of Halpern and O'Connor (2000). In fact, pointing at the preferred stimulus does not require the same processes as those involved in translating one's affective attitude towards a stimulus on a rating scale. Rating might involve more complex processes, such as understanding the correspondence between the scale and the feeling intensity, metacognitive processes, maintaining the choice in working memory long enough to make a rating, etc. All these processes might be too complex or impaired in AD patients, leading to a biased estimation of the mere exposure effect. On the contrary, pointing is a relatively easy and direct way to express the preference. In addition, both studies also differ in the number of exposed stimuli (19 in Winograd et al.'s study vs. 8 in Halpern and O'Connor's study) as well as in the encoding procedure: Halpern and O'Connor used only two presentations for each melody, and only one encoding task (judging the melody speed), while Winograd et al. used three five-second duration exposures for each face with three different judgments about different face features (eye colour, hair colour, and age). This last condition certainly leads to a more precise perceptual encoding. Another important contrast between these studies is the type of processing involved as a function of the stimulus type. A face presented during $5 \mathrm{sec}$ can be processed as a whole, with all parts being presented in the same time. On the contrary, a $5 \mathrm{sec}$ melody is, by definition, sequential and its constituting parts cannot be processed 
simultaneously, but in succession. It is, therefore, possible that the AD patients' deficit in melodic preference was the consequence of either the demands associated to the preference judgment task or the characteristics of the encoding condition. Finally, a diffrence of age existed between the AD patients groups $(78.7+/-6.2$ in Halpern \& O'Connor's study and 69 +/- 10 in Winograd et al.'s study). Even if both groups were relatively equivalent regarding the dementia severity, the age difference between samples might have been a final factor involved in the discrepancy of results between of both studies.

To summarise, the evidence concerning the mere exposure effect in Alzheimer's disease is rather sparse, especially when compared to the numerous studies devoted to the perceptual or conceptual expressions of implicit memory (see Fleischman and Gabrieli, 1998; Meiran and Jelikic, 1995). Furthermore, the only two existing studies have provided contradictory results, and their results are not easily comparable. It is, therefore, hard to characterise the pattern of success and failure of $\mathrm{AD}$ patients on the mere exposure effect, when considering the differences between these studies.

Finally, a last problem should be mentioned concerning the Winograd et al. (1999) study. Indeed, in their research, preference judgments (Experiment 1) and recognition scores (Experiment 2) were obtained in separate groups of $\mathrm{AD}$ patients. Both groups were not statistically different in age, education, or dementia severity, but, because of the neuropsychological heterogeneity of $\mathrm{AD}$, the observed dissociation between a preserved mere exposure effect for faces and an impaired face recognition performance should be interpreted with caution. In addition, in Experiment 2, the healthy control subjects were matched for age with the AD patients but had completed more years of education, which also makes the AD patients' recognition deficit questionable.

Considering all these problems, it clearly appears that further studies are needed in order to better characterise the mere exposure effect in AD. The purpose of the present 
research was to re-examine the mere exposure effect for unfamiliar faces in AD patients. Similarly to the Winograd et al.'s study, we used preference and recognition tasks with a same format (forced-choice judgments). However, contrary to that study, preference judgment and recognition performance were explored in the same group of patients and control subjects. Furthermore, very short exposure times and a unique encoding task (age estimation) were used in order to make explicit recognition especially difficult and consequently, to optimise the possible dissociation between recognition performance and preference judgments. Despite these modifications in the encoding condition, we expected the mere exposure effect to be preserved in AD patients as it was in Winograd et al.'s study. Indeed, mere exposure effect does not seem to be favoured by long exposure duration or detailed perceptual encoding (Bornstein, 1989).

\section{Method}

\section{$\underline{\text { Participants }}$}

Two groups of subjects participated in this study: patients with dementia of the Alzheimer type (AD) and normal elderly subjects. The selected AD group consisted of fourteen subjects (four males and ten females) who met the NINCDS-ADRDA criteria for probable Alzheimer's disease (McKhann, Drachman, Folstein et al., 1984). Table 1 displays the participants' characteristics. All patients had suffered from progressive worsening of memory problems for at least six months. The diagnosis was based on general medical, neurological and neuropsychological examinations. Exclusion criteria were a premorbid history of major psychiatric or neurological illness, drug or alcohol abuse. Their vision was normal or corrected. CT scans showed mild atrophy, at most. Patients' ranged was from 107 to 138 on the Mattis dementia rating scale (DRS; Mattis, 1973) and from 12 to 26 on the MMSE (Folstein, Folstein and McHugh, 1975). 
Fourteen normal elderly subjects, matched for age, sex and educational level, served as controls. The normal controls were non-institutionalised, alert, and submitted to the same exclusion criteria as the AD group. As shown in Table 1, these control subjects did not differ from AD patients as to their age and their schooling level. All controls had a total score superior to 130 on the DRS, which constitutes a cut-off score to discriminate normal aging from dementia (Monsch, Bondi, Salmon et al., 1995). Their average scores were between 131 and 144 on the DRS, and between 26 and 30 on the MMSE. As shown in Table 1, overall performance was significantly lower for AD patients than for controls on the DRS and the MMSE.

\section{Insert Table 1 about here}

\section{$\underline{\text { Materials }}$}

Stimuli were 60 black-and-white photographs of unfamiliar men's faces. Selected photographs were chosen from a sample of 126 photographs. Photographed people ranged from 20 to 50 years of age. None of the presented faces had a beard, moustache, long hair, glasses or other distinctive features. The facial expression was neutral. Faces had been previously photographed under even conditions, that is, the same artificial lighting with the same lighting angle, the same face orientation ( $45^{\circ}$ profile), and the same distance between face and camera. The body, the neck and the background were erased using the Adobe Photoshop Software (Adobe Systems, Inc.).

In a preliminary phase, we prepared 15 equally likeable pairs of faces that could be used in the preference test. Firstly, each face of the 126 photographs was rated by 20 undergraduate students on a visual analogue scale of "likeability" $(0 \mathrm{~cm}=$ very dislikeable, $10 \mathrm{~cm}=$ very likeable). Faces were then paired according to their average likeability rating. 50 pairs were created in such a way that both members of each pair were approximately equally rated on the 
likeability scale. The 50 pairs of faces were randomly divided into two sets (set A and B) in such a way that each set contained one member from each pair. Secondly, each pair of faces was rated by 20 other undergraduate students in a forced-choice preference judgment. For this experiment, we kept the pairs in which both members were selected equally often. As a result of this two-step pre-test selection, 15 pairs of equally-likeable faces were included in this study. Faces A and B in the selected pairs had a mean score of 0.50 in the preference judgment $(\underline{\mathrm{SD}}=0.02$, range: 0.47 to 0.53$)$. Half of the subjects were presented with set $\mathrm{A}$ of stimuli as the target and set B as distractors. The other half of the subjects were presented with the reverse design.

Each target in set A and set B was paired with a new face by apparent age and general attractiveness for creating 30 new target-distractor pairs for the recognition test.

All stimuli were presented on a 14" screen approximately $70 \mathrm{~cm}$ from subject. Faces were about $11 \mathrm{~cm}$ in height and $8 \mathrm{~cm}$ in width. They appeared on a black background, which yielded a pronounced figure-ground contrast. Each picture was made equivalent regarding the file size (100ko) and was presented by using the MEL 2.0 Professional software (Psychology Software Tools, Inc.) on a single 486/66 MHz PC with 16 mega of RAM, to every subject.

Conditions of low room illumination were used throughout the experiment. Instructions were provided in these conditions, which allowed dark adaptation during a few minutes (over five minutes for every paticipant).

\section{$\underline{\text { Procedure }}$}

Exposure phase. Patients and controls were tested in individual sessions. Subjects were told that the study involved "face perception", and that they were going to see 15 faces, five times. They were informed that stimuli would be presented very briefly and would be difficult to see. After viewing three examples, they were asked to estimate the age of the target faces by responding less than 30 years old or more than 30 years old. Considering the brevity of 
presentation, the task was rather difficult. However, this task allows to ensure that the subject stays concentrated throughout the presentation. No mention was made of any subsequent memory test.

Subjects were then presented with 15 faces, five times each, in five random orders of 15. Each study stimulus was presented at the centre of the screen for $17 \mathrm{~ms}$, preceded by a fixation point presented for $1500 \mathrm{~ms}$. The interval between the fixation point and the onset of the stimulus was $400 \mathrm{~ms}$. Each stimulus was followed by a $1000 \mathrm{~ms}$ interval. The interstimulus interval was thus equal to $2900 \mathrm{~ms}$.

In the exposure phase, three patients and three controls reported that they could not perceive faces sufficiently to make an age judgment and to respond less than or more than 30 years old (their inability to make this age judgment was consistent across trials). Indeed, they reported being aware that faces had appeared on the screen, but without being able to process them because they disappeared too soon. In this case, subjects were told simply to look at the screen.

Immediately following the exposure session, a distractor verbal task (the DRS, Mattis, 1973) was administered during 15 minutes. Since, the DRS had already been administered to five $\mathrm{AD}$ patients in the context of a neuropsychological examination in the previous month, a verbal recognition task of the same duration was used for these patients.

Forced-choice preference judgment. Subjects were presented with 15 pairs of faces containing a previously shown target face and a novel distractor face. Both faces were presented simultaneously to the subject. Each pair was presented for $1 \mathrm{sec}$, followed by a selfspaced interstimulus interval of approximately 1 to 2 seconds. The side of the screen in which the target stimulus was displayed was counterbalanced and randomised over trials. Subjects were asked to examine each pair and to point to the face they liked best. 
$\underline{\text { Recognition forced-choice judgment. Fifteen pairs containing the target face and a }}$ distractor face were displayed. Subjects were asked to point to the stimulus that they had seen during the exposure phase. The procedure and exposure duration were identical to that used in preference judgment.

All subjects were first presented with the preference judgment task, followed by recognition judgment. This order was used to limit the risk that subjects would suspect the preference judgment to be a memory test.

\section{Results}

The mean proportion of target faces selected in the recognition and preference condition are presented in Figure 1. It must be noted that analyses carried out on all subjects or on a reduced group excluding the three patients who were unable to process the faces, gave the same results. Therefore, for the sake of clarity, we only present here the analyses performed on the entire groups, except when necessary.

\section{INSERT FIGURE 1 ABOUT HERE}

The mean proportion (with $\underline{\mathrm{SDs}}$ in parentheses) of target faces preferred was $.58(.12)$ for controls and $.57(.12)$ for AD patients (see Figure 1). Subjects selected targets at greater than chance (estimated at .50), for controls $[\underline{t}(13)=2.293]$ and for AD patients $[\underline{t}(13)=$ 2.245], one-tailed, $\underline{\mathrm{P}}<.025$.

Regarding recognition, one $\mathrm{AD}$ patient was excluded from the analyses because he kept on making a preference judgment instead of recognition, as shown by his verbal comments (e.g., the patient kept saying: 'I don't like this one'). The mean proportion of target faces correctly recognized by controls was .65 (.07). This mean was significantly greater than $.50, \underline{\mathrm{t}}(13)=7.763$, one-tailed, $\underline{\mathrm{P}}<.001$. AD patients' proportion of correctly recognized faces 
was $.53(.14)$, which was not significantly different from $.50, \underline{t}(12)=.8811$, one-tailed, $\mathrm{P}>$ .05 .

A 2 (groups) x 2 (type of test: preference vs recognition) ANOVA was then carried out. The analysis revealed a significant interaction $[\underline{F}(1,25)=6.04, \underline{P}=.021]$ but no effect of group $(\underline{\mathrm{P}}=.14)$ nor effect of type of test $(\underline{\mathrm{P}}=.77)$. Planned comparisons detailed this interaction by indicating no significant difference in the preference effect between the groups $[\underline{F}(1,25)=$ $.034, \underline{\mathrm{P}}=.854]$ despite a significant difference in recognition $[\underline{\mathrm{F}}(1,25)=7.747, \underline{\mathrm{P}}=.01]$. When the participants who had problems in processing the faces were excluded from the analysis, the ANOVA revealed an interaction only close to significance $[\underline{F}(1,19)=3.37, \underline{P}=$ .082] but no effect of group nor type of test effect $(\underline{\mathrm{P}}>$.05). Planned comparisons still indicated no significant difference in the preference effect between the groups $[\underline{F}(1,19)=$ $.042, \underline{\mathrm{P}}=.84]$ despite a significant difference in recognition $[\underline{\mathrm{F}}(1,19)=5.596, \underline{\mathrm{P}}=.029]$. Here, the difference observed for the two-way ANOVA might be explained by the reduced number of subjects and, thus, by a lack of power.

\section{Discussion}

Despite a striking impairment in recognition memory, the AD patients demonstrated a normal mere exposure effect. These results not only confirm but also amplify the findings of Winograd et al. (1999). Indeed, contrary to Winograd et al., the dissociation between explicit memory performance and the mere exposure effect was obtained in the same groups of AD patients and control subjects. Another difference with the Winograd et al. study concerned the encoding condition: we used a very brief exposure duration and only one encoding task (age estimation), while Winograd et al. used three different encoding tasks and a $5 \mathrm{sec}$ exposure duration. The preservation of the mere exposure effect in these encoding conditions suggests that the existence of this effect in $\mathrm{AD}$ patients does not depend on long exposure duration or detailed perceptual encoding. 
Finally, the preservation of the mere exposure effect was observed in the presence of a chance level recognition performance, which suggests that these two types of phenomenon are functionally independent. These findings converge on a considerable number of experiments that have obtained a robust exposure effect for visual stimuli presented so briefly at study that they were not recognized by normal young subjects on a subsequent memory test (e.g. Bornstein, Leone and Galley, 1987; Kunst-Wilson and Zajonc, 1980; Mandler, Nakamura and Van Zandt, 1987; Seamon, Brody and Kauff, 1983a, 1983b).

Different hypotheses may be proposed to account for the finding of affective preference without recognition in AD patients (see Seamon, McKenna and Binder, 1998, for a discussion of different theories of the mere exposure effect). According to Zajonc (2000), the nonconscious mere exposure effect occurs because affective processing can proceed independently of cognitive processing. The affective responses would normally precede the cognitive processing and take place virtually without the contribution of any cognitive input. In support of this hypothesis of independence between affective and cognitive processing, Zajonc cited several findings suggesting that different anatomical structures are involved in both of these processes. According to this point of view, a large body of research has linked the amygdala to memory processes for emotional events, the processing of certain emotional expressions, the preference judgment for visual stimuli and the conditioned preference (see Adolphs and Tranel, 1999; Fine and Blair, 2000; Johnsrude, Owen, White et al., 2000). With regard to $\mathrm{AD}$ patients, studies on histopathological changes or on the basis of magnetic resonance imaging morphometry indicate that the degeneration of the amygdala may occur very early in the course of the disease (e.g. Chow and Cummings, 2000; Maunoury, Michot, Caillet et al., 1996; Van Hoesen, 1997). However, the specific contribution of the amygdala in the mere exposure effect development has never been investigated. Moreover, our findings, 
which showed a preserved mere exposure effect in $\mathrm{AD}$ patients, rather suggest that this effect does not depend on the amygdala.

Another interpretation has been offered for the mere exposure effect. A number of researchers have argued that a feeling of familiarity resulting from perceptual fluency plays an important role in the mere exposure effect (e.g., Bornstein and D'Agostino, 1992; Seamon et al., 1983a). Perceptual fluency can be defined as the facilitation of stimulus encoding as a result of perceptual encoding practice (Jacoby and Dallas, 1981). With regard to the mere exposure effect, this ease of processing has been hypothesised to serve as the basis for preference judgment (Bornstein and D’Agostino, 1992). Thus, when asked to choose between previously shown and novel stimuli in a preference judgment task, subjects may prefer previously exposed stimuli because they are easier to process (Seamon et al., 1983a). Consistent with this view, Reber, Winkielman and Schwarz (1998) observed that preference for neutral stimuli can be enhanced by manipulations of perceptual fluency (e.g., changes in figure-ground contrast), independently of stimulus repetition. At the same time, the feeling of familiarity resulting from ease of processing also serves as a basis for recognition memory (e.g., Verfaellie and Cermak, 1999). Consequently, the perceptual fluency could be used as a cue for recognition and as a basis for preference judgment. However, one could then ask why, in current findings, AD patients' performances differ significantly from chance in preference but not in recognition. From this point of view, these findings might suggest a dissociation between the familiarity which might be involved in the mere exposure effect and that involved in recognition. Familiarity thus appears to be multidetermined (Verfaellie and Cermak, 1999). Indeed, dissociations between familiarity processes in recognition and implicit perceptual memory can be observed (Wagner, Gabrieli and Verfaellie, 1997).

More recently, Seamon and his collaborators (Seamon, Williams, Crowley et al., 1995; Seamon, Ganor-Stern, Crowley et al., 1997; Seamon and Delgado, 1999) suggested that the 
mere exposure effect, like perceptual priming, can be mediated by a presemantic perceptual representation system (PRS) that codes the information about the structure and form of objects. PRS consists of modality-specific subsystems of memory like, for example, a structural description system which contains representations of structural relations among the component parts of three-dimensional objects. Thus, subjects would prefer previously presented stimuli more than non-presented stimuli because the former have an available representation in the PRS that later underlies a perceptual fluency. PRS is mediated by modality-specific cortices (Schacter, 1994) and evidence for a neuroanatomical basis of perceptual priming in visual modality comes from imaging studies that have demonstrated extrastriate cortices activation (Badgaiyan, 2000; Henson, Shallice and Dolan, 2000; see also Nyberg, 2000). Given that the perceptual priming seems to be intact in AD patients (for a review, Fleischman and Gabrieli, 1998), it is plausible that occipital regions subserve this intact performance in $\mathrm{AD}$ patients. In this prospect, it has been shown that the neurofibrillary tangles increase in $\mathrm{AD}$ patients as one moves from primary sensory (area 17) to extrastriate areas (area 19) (Lewis, Campbell, Terry et al., 1987), these latter areas remain, however, considerably less prone to tangle formation than multimodal association cortices and limbic and higher order associations areas (Arnold, Hyman, Flory et al., 1991; Pietrini, Furey, Alexander et al., 1999). In addition, Bäckman, Almkvist, Nyberg et al. (2000) recently observed in $\mathrm{AD}$ patients as well as in control subjects a correlation between perceptual priming performance and activity in area 19, although the type of activation pattern was different in both groups. In this way, the current findings of a mere exposure effect for unfamiliar faces in $\mathrm{AD}$ patients may reflect the relative sparing of the PRS. Relevant to this context are the findings of Halpern and O'Connor (2000), who found no effect for melodies. It is possible that, given the domain-specific nature of PRS, this discrepancy can reflect a 
dissociation between a preserved subsystem PRS dedicated to faces and an impaired subsystem for processing melodies in Alzheimer'disease.

Finally, we must remain cautious about the conclusion that the mere exposure effect for unfamiliar objects is entirely preserved in Alzheimer' disease Firstly, it is possible that, in our study, like in that of Winograd et al. (1999), the significant but small effect did not enable the observation of an amplitude difference between groups. Indeed, although it is significant, the preference for old faces observed in these studies is small. In this context, it seems that faces are not the most sensitive materials for exploring the mere exposure effect. Indeed, faces have a specific status since they are very important social stimuli for humans and bear high emotional value. Also, the monitoring of the emotional valence seems to be extremely rapid and automatic (Ledoux, 1995; Pizzagali, Regard and Lehmann, 1999). Therefore, it is possible that the automaticity and rapidity of these mechanisms prevent a more larger expression of the mere exposure effect. Secondly, in our experiment, the delay between the study and test phase was rather short. Seamon et al. (1983b) found a preference effect in normal subjects over long delays. Thus, an impaired mere exposure effect might be expressed as a mere exposure effect that declines faster over time in $\mathrm{AD}$ patients than in controls. Finally, as noted by Halpern and O'Connor (2000) and Verfaellie et al. (2000), a very small number of studies have been carried out in the auditory implicit memory domain, with AD patients. Furthermore, given the discrepancy between the mere exposure effect results obtained in $\mathrm{AD}$ patients in the visual and auditory modality, and between the impaired exposure effect for melodies in Hapern and O'Connor's study and preserved priming for auditorily presented words in the Verfaellie et al. study, a further investigation of preference effect in different modalities with a same procedure is necessary. However, materials, other than melodies, could be fruitfully used in auditory modality exploration. 


\section{Acknowledgements}

We gratefully acknowledge the participation of patients from the Day Care Center, CHU of Liège, a multidisciplinary clinical and research unit that depends on the Departments of Neurology (prof. Moonen) and of Neuropsychology (Prof. Van der Linden), University of Liège. We thank Philippe Marczewski for helping with the preparation of this article. 


\section{Author's notes}

Sylvie Willems, and Martial Van der Linden, Unité de Psychopathologie Cognitive, Université de Genève, Switzerland ; Stéphane Adam, and Martial Van der Linden, Service de Neuropsychologie, Université de Liège, Belgium.

Adam Stéphane is supported by the Grant 98/03 - 215 of the Government of the French-Language Community of Belgium (Actions de Recherche Concertées).

Reprint request should be sent to Sylvie Willems, Sylvie.willems@ pse.unige.ch, Unité de Psychopathologie Cognitive, University of Geneva, Bv. Pont d'Arve, 40, CH-1205 Geneva, Switzerland. 


\section{References}

AdolPhS, R., and Tranel, D. Preferences for visual stimuli following amygdala damage. Journal of Cognitive Neuroscience, 11: 610-616, 1999.

Arnold, S.E., Hyman, B.T., Flory, J., Damasio, A.R., and Van Hoesen, G.W. The topographical and neuroanatomical distribution of neurofibrillary tangles and neuritic plaques in cerebral cortex of patients with Alzheimer's disease. Cerebral Cortex, 1: 103-116, 1991.

Bäckman, L., Almkvist, O., Nyberg, L., and Andersson, J. Functional changes in brain activity during priming in Alzheimer's disease. Journal of Cognitive Neuroscience, 12: 134-141, 2000.

BADGAIYAN, R.D. Neuroanatomical organization of perceptual memory: An fMRI study of picture priming. Human Brain Mapping, 10: 197-203, 2000.

BORNSTEIN, R.F. Exposure and affect: Overview and meta-analysis of research, 19681987. Psychological Bulletin, 106: 265-289, 1989.

Bornstein, F.R., and D'Agostino, P.R. Stimuli recognition and the mere exposure effect. Journal of Personality and Social Psychology, 4: 545-552, 1992.

Bornstein, R.F., LeONE, D.R., and GALLEY, D.J. The generalizability of subliminal mere exposure effects: Influence of stimuli perceived without awareness on social behaviour. Journal od Personality and Social Psychology, 53: 1070-1079, 1987.

Chow, T.W., and Cummings, J.L. The amygdala and Alzheimer's disease. In J.P. Aggleton (Ed.), The amygdala: A functional analysis. Oxford: Oxford University Press, 2000, pp. 656-680. 
FINE, C., and BLAIR, R.J.R. The cognitive and emotional effect of amygdala damage. Neurocase, 6: 435-450, 2000.

Fleischman, D.A., and GABRIELI, J.D. Repetition priming in normal aging and Alzheimer's disease: a review of findings and theories. Psychology and Aging, 13: 88-119, 1998.

Folstein, M., Folstein, S., and Mc Hugh, P.R. Mini-mental state: A practical method for grading the cognitive state of patients for clinician. Journal of Psychiatric Research, 12: 189-198, 1975.

GREVE, K.W., and BAUER, R.M. Implicit learning of new faces in prosopagnosia: an application of the mere-exposure paradigm. Neuropsychologia, 28: 1035-1041, 1990.

HALPERn, A.R., and O'CONNOR, M.G. Implicit memory for music in Alzheimer's disease. Neuropsychology, 14: 391-397, 2000.

HARrisson, A.A. Mere Exposure. In L. Berkowitz (Ed.), Advances in Experimental Social Psychology, vol. 10. New York: Academic Press, 1977, pp. 39-83.

Henson, R., Shallice, T., and Dolan, R. Neuroimaging evidence for dissociable forms of repetition priming. Science, 287: 1269-1272, 2000.

JACOBY, L.R., and DALLAS, M. On the relationship between autobiographical memory and perceptual learning. Journal of Experimental Psychology: General, 3: 306-340, 1981.

Johnson, M.K., KIM, J.K., and RISSE, G.. Do alcoholic Korsakoff's syndrome patients acquire affective reactions? Journal of Experimental Psychology: Learning, Memory, and Cognition, 11: 22-36, 1985. 
Johnson, M.K., and Multhaup, K.S. Emotion and MEM. In S.-A. Christianson (Ed.), The Handbook of Emotion and Memory. Hillsdale, NJ: Erlbaum, 1992, pp. 33-66.

Johnsrude, I.S., Owen, A.M., White, N.M., Zhao, W.V., and Bohbot, V. Impaired preference conditioning after anterior temporal lobe resection in humans. Neuroscience, 20: 2649-2656, 2000.

Kinlstrom, J.F., Mulvaney, S., Tobias, B.A., and Tobis, I.P. The emotional unconscious. In E. Eich, J.F. Kihlstrom, G.H. Bower, J.P. Forgas, and P.M. Niedenthal (Eds.), Cognition and emotion. Oxford: Oxford University Press, 2000, pp. 30-86.

KUnST-WILSON, W.R., and ZAJONC, R.B. Affective discrimination of stimuli that cannot be recognized. Science, 207: 557-558, 1980.

LEDoux, J.E. Emotion: clues from the brain. Annual Review of Psychology, 46: 209$235,1995$.

Lewis, D.A., CAMPBell, M.J., TERry, R.D., and Morrison, J.H. Laminar and regional distributions of neurofibrillary tangles and neuritic plaques in Alzheimer's disease: A quantitative study of visual and auditory cortices. Journal of Neurosciences, 7: 1799-1808, 1987.

Mandler, G., NAKAmURA, Y., and VAn ZANDT, B.J. Nonspecific effects of exposure on stimuli that cannot be recognized. Journal of Experimental Psychology: Learning, Memory, and Cognition, 13: 646-648, 1987.

MAtTIS, S. Dementia Rating Scale. Windsor: NFER-Nelson, 1973.

Maunoury, C., Michot, J.L., Caillet, H., Parlato, V., Leroy-Willig, A., Jehenson, P., Syrota, A., and Boller, F. Specificity of temporal amygdala atrophy in 
Alzheimer's disease: Quantitative assessment with magnetic resonance imaging. Dementia, 7: 10-14, 1996.

McKhann, G., Drachman, D., Folstein, M., Katzman, R., Price, D., and Stadlan, E.M. Clinical diagnosis of Alzheimer's disease: Report of the NINCDS-ADRDA work group under the auspices of department of health and human services task force on Alzheimer's disease. Neurology, 34: 939-944, 1984.

MEIRAN, N., and JELICIC, M. Implicit memory in Alzheimer's disease: A meta-analysis. Neuropsychology, 9: 291-303, 1995.

Monsch, A.U., Bondi, M.W., Salmon, D.P., Butters, N., Thal, L.J., Hansen, L.A., Wiederholt, W.C., CAHn, D.A., and KLAUber, M.R. Clinical validity of the Mattis dementia rating scale in detecting dementia of the Alzheimer type. Archives of Neurology, 52: 899-504, 1995.

NyBERG, L. Perceptual priming and extrastriate cortex: Consensus and controversy. Human Brain Mapping, 10: 195-196, 2000.

Pietrini, P., Furey, M.L., Alexander, G.E., Mentis, M.J., DAni, A., GuAZzelli, M., RAPOPORT, S.I., and SCHAPIRO K.B. Association between brain functional failure and dementia severity in Alzheimer's disease: Resting versus stimulation PET study. American Journal of Psychiatry, 156: 470-473, 1999.

PizZagalli, D., Regard, M. and Lehmann, D. Rapid emotional face processing in the human right and left brain hemispheres: An ERP study. Neuroreport, 10: 2691-2698, 1999.

Reber, R., Winkielman, P., and SchwarZ, N. Effects of perceptual fluency on affective judgments. Psychological Science, 9: 45-48, 1998. 
Redington, K., VolPe, B.T., and GaZZANiGA, M.S. Failure of preference formation in amnesia. Neurology, 34: 536-538, 1984.

SCHACTER, D.L. Priming and multiple memory systems: Perceptual mechanisms of implicit memory. In D.L. Schacter and E. Tulving (Eds.), Memory systems, 1994. Cambridge, MA: MIT, 1994, pp.233-268.

SEAMON, J.G., Brody, N., and KAUFF, D.M. Affective discrimination of stimuli that are not recognized: Effects of shadowing, masking, and cerebral laterality. Journal of Experimental Psychology: Learning, Memory, and Cognition, 9: 544-555, 1983a.

SEAmon, J.G., Brody, N., and KAUFF, D.M. Affective discrimination of stimuli that are not recognized II: Effects of delay between study and test. Bulletin of the Psychonomic Society, 21: 187-189, 1983b.

SeAmon, J.G., and Delgado, M.R. Recognition memory and affective preference for depth-rotated solid objects: Part-based structural descriptions may underlie the mere exposure effect. Visual Cognition, 6: 145-164, 1999.

Seamon, J.G., Ganor-Stern, D., Crowley, M.J., Wilson, S.M., Weber, W.J., O'Rourke, C.M., and MAhoney, J.K. A mere exposure effect for transformed threedimensional objects: Effects of reflection, size, or color changes on affect and recognition. Memory \& Cognition, 25: 367-374, 1997.

Seamon, J.G., McKenna, P.A., and Binder, N. The mere exposure effect is differentially sensitive to different judgment tasks. Consciousness and Cognition, 7: 85-102, 1998.

Seamon, J.G., Williams, P.C., Crowley, M.J., Kim, I.J., LAnger, S.A., Orne, P.J., \& WISHENGRAD, D.L. The mere exposure effect is based on implicit memory: Effect of stimulus 
type, encoding conditions, and number of exposures on recognition and affect judgments. Journal of Experimental Psychology: Learning, Memory, and Cognition, 21: 711-721, 1995.

VAN Hoesen, G.W. Ventromedial temporal lobe anatomy, with comments on Alzheimer's disease and temporal injury. Journal of Neuropsychiatry and Clinical Neuroscience, 9: 331-341, 1997.

Verfaellie, M., and Cermak, L.S. Perceptual fluency as a cue for recognition judgments in amnesia. Neuropsychology, 13: 198-205, 1999.

Verfaellie, M., Keane, M.M., and Johnson, G. Preserved priming in auditory perceptual identification in Alzheimer's disease. Neuropsychologia, 38: 1581-1592, 2000.

Wagner, A., Gabrieli, J.D.E., and Verfaellie, M. Dissociations between familiarity processes in explicit recognition and implicit perceptual memory. Journal of Experimental Psychology: Learning, Memory, \& Cognition, 23: 305-323, 1997.

Winograd, E., Goldstein, F.C., Monarch, E.S., Peluso, J.P., and Goldman, W.P. The mere exposure effect in patients with Alzheimer's disease. Neuropsychology, 13: 41-46, 1999.

ZAJONC, R.B. Attitudinal effects of mere exposure. Journal of Personality and Social Psychology Monograph Supplement, 9: 1-27, 1968.

ZAJONC, R.B. Feeling and thinking: Closing the debate over the independence of affect. In J.P. Forgas (Ed.), Feeling and thinking: The role of affect in social cognition. Cambridge: Cambridge University Press, 2000, pp.31-58. 
Table 1

Participants Characteristics

\begin{tabular}{lccc}
\hline & AD patients & Controls & $t(26)$ \\
\hline Female & 10 & 10 & \\
Male & 4 & 4 & -0.52 \\
Age (years) & & & \\
M & 75.5 & 74.3 & \\
SD & 5.7 & 6.6 &
\end{tabular}

Education (years)

0.64

M

10.9

11.9

SD

4.2

4

DRS

M

120.1

139.2

$7.01 *$

SD

9.1

4.3

Range

$107-138$

$131-144$

MMSE

$$
\mathrm{M}
$$

20.7

28.8

$7.26^{*}$

SD

1.4

Range

$22-26$

$26-30$

Note. For both groups, $\underline{\mathrm{N}}=14$; DRS = Mattis Dementia Rating scale (Coblentz et al., 1973); MMSE = Mini-Mental State Examination (Folstein, Folstein, \& McHugh, 1975); $\mathrm{AD}=$ Alzheimer's disease .

$* \underline{\mathrm{P}}<.001$ 


\section{Figure LEGENDS}

Figure 1: proportion of target faces selected 
$\underline{\text { Figure } 1}$

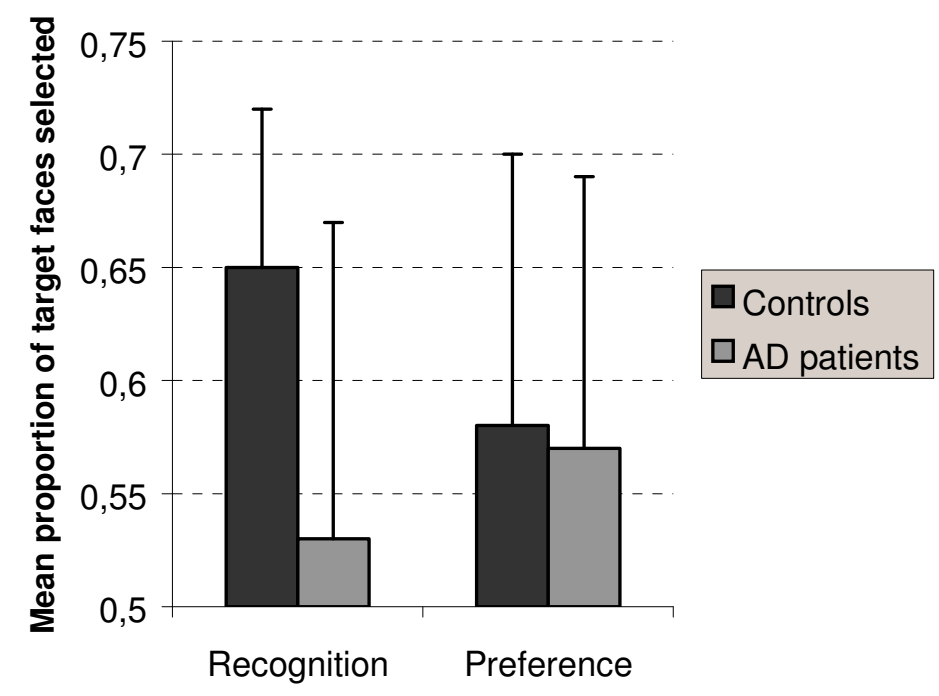

